\title{
Definition of Turbulent Boundary-Layer with Entropy Concept
}

\author{
Zhao Rui $^{1}$, Rong Jili ${ }^{1}$, LI Haibo ${ }^{2}$ and Ren Fang ${ }^{2}$ \\ ${ }^{1}$ School of Aerospace Engineering, Beijing Institute of Technology, Beijing 100191, P. R. China \\ ${ }^{2}$ Beijing Institute of Structure and Environment Engineering, Beijing 100076, P.R. China
}

\begin{abstract}
The relationship between the entropy increment and the viscosity dissipation in turbulent boundary-layer is systematically investigated. Through theoretical analysis and direct numerical simulation (DNS), an entropy function $f_{\mathrm{s}}$ is proposed to distinguish the turbulent boundary-layer from the external flow. This approach is proved to be reliable after comparing its performance in the following complex flows, namely, low-speed airfoil flows with different wall temperature, supersonic cavity-ramp flow dominated by the combination of free-shear layer, larger recirculation and shocks, and the hypersonic flow past an aeroplane configuration. Moreover, $f_{\mathrm{s}}$ is deduced from the point of energy, independent of any particular turbulent quantities. That is, this entropy concept could be utilized by other engineering applications related with turbulent boundary-layer, such as turbulence modelling transition prediction and engineering thermal protection.
\end{abstract}

\section{Introduction}

Entropy, in addition to energy, is a central physical quantity in thermodynamics. It serves as a measure of the irreversibility of a process and a criterion describing the thermal equilibrium of a system. It also represents the degree of disorder within a system [1]. Because of its general features, the entropy concept has been extended to many other fields, such as thermodynamic optimization, information theory and computational techniques [2]-[6].

As for the turbulent flow, McEligot et al. [7] concluded that about two-thirds or more of entropy generation occurs in the turbulent boundary-layer. Then, Zhao et al. attempted to denote the turbulent boundarylayer by the entropy concept. Through Direct Numerical Simulation (DNS) of supersonic compression ramp flows, they found that the value of entropy is much larger in the boundary-layer and keeps decreasing monotonously in the wall normal direction [8]. Based on this character, they revised the length scale of Baldwin-Lomax model, enhancing its robustness in simulating supersonic complex flows. Although these achievements indicate another latent capacity of entropy, the definition of entropy expression for turbulent boundary-layer is still not comprehensive.

This paper continues the previous work, and tries to extend this entropy concept from both the theoretical and numerical aspects. We hope this investigation here could be served as another approach for turbulence modeling, transition prediction, etc., where the calculated turbulent boundary-layer is always necessary.

\section{Entropy expression}

Virtually all flows of practical engineering interest are turbulent. Due to the irregular motion of turbulence, the transportations of mass, momentum and energy are enhanced, while extra energy is dissipated. In the near wall region, as for the fierce turbulent fluctuation and wall frication, a portion of mechanical energy is irreversibly transformed into internal energy, i.e. the entropy increase. It should be noted that a similar process also occurs due to the wall heat flux or the deceleration of flows where shock waves appear.

For the perfect gas model, the relation of entropy increment and energy change is revised by

$$
\mathrm{d} s=\frac{\mathrm{d} q}{T}
$$

where $s$ is entropy, $q$ is the heat transfer, and $T$ is the local temperature. This form of the definition is different from the usual one [9] in two aspects: (1) $q$ is the actual heat added on the system, no longer limited to the one caused by the reversible process only. (2) Correspondingly, the entropy increment $d s$ is proportional to the exact differential $d q$.

With the conservation law of energy, also called First Law of Thermodynamic

$$
\mathrm{d} q=\mathrm{d} u+p \mathrm{~d}\left(\frac{1}{\rho}\right)
$$

substituted into Eq. (1), we can finally get 


$$
\mathrm{d} s=c_{V} \mathrm{~d}\left(\ln \frac{p}{\rho^{\gamma}}\right)
$$

where $u=c_{V} T$ is the internal energy, $c_{V}=R /(\gamma-1)$ is the specific heat at constant volume, $R$ is the gas constant, $\gamma=1.4$ is the specific heat ratio, and $p$ and $\rho$ are the local pressure and density, respectively. As Eq. (3) shows, $s$ is proportional to $p / \rho^{\gamma}$. We define entropy in this research as

$$
s=\frac{p}{\rho^{\gamma}}
$$

As aforementioned, the increment of $s$ is proportional to the heat transfer $q$, no matter where the heat comes from (Eq. (1)). However, in order to employ the increment of $s$ to discern the boundary-layer, the first step we should do is to separate the heat caused by viscosity dissipation from the total, especially from the wall heat flux due to both are mainly produced in the near wall region.

\subsection{Process I: Entropy increment produced by viscosity dissipation}

One of the commonly used temperature-velocity relationships for zero-pressure gradient boundary layers is Walz's equation [10].

$$
\frac{T}{T_{\infty}}=\frac{T_{\mathrm{w}}}{T_{\infty}}+\frac{T_{\mathrm{r}}-T_{\mathrm{w}}}{T_{\infty}}\left(\frac{u}{u_{\infty}}\right)+\frac{T_{\infty}-T_{\mathrm{r}}}{T_{\infty}}\left(\frac{u}{u_{\infty}}\right)^{2}
$$

in which the subscript $\infty$ denotes the free-stream values and the recovery temperature $T_{\mathrm{r}}=T_{\infty}\left(1+r(\gamma-1) M a_{\infty}{ }^{2} / 2\right)$ with the recovery factor $r=0.9$.

Differencing each side at the wall by multiplying the heat conductivity coefficient $k$, we can get the Reynolds analogy relationship

$$
-\left.k \frac{\partial T}{\partial y}\right|_{\mathrm{w}}=\frac{T_{\mathrm{w}}-T_{\mathrm{r}}}{(\gamma-1) \operatorname{Pr}_{\mathrm{t}} M a_{\infty}^{2} T_{\infty}} \times\left. u_{\infty} \mu \frac{\partial u}{\partial y}\right|_{\mathrm{w}}
$$

where $k=\mu c_{p} / P r_{t}, \quad c_{p}=R \gamma /(\gamma-1)$ is the specific heat at constant pressure, and $\operatorname{Pr}_{t}=0.9$ is the turbulent Prandtl number.

Obviously, the left term in Eq. (6) is the heat caused by the wall heat flux $\left(\mathrm{Q}_{2}\right)$ and the second term on the right is approximately the heat produced by the viscosity dissipation of kinetic energy $\left(Q_{1}\right)$. Thus, in the near wall region, we may separate the sources of heat by defining a new parameter $\sigma$,

$$
\sigma=\frac{T_{\mathrm{w}}-T_{\mathrm{r}}}{(\gamma-1) \operatorname{Pr}_{\mathrm{t}} M a_{\infty}^{2} T_{\infty}} \approx \frac{\mathrm{Q}_{2}}{\mathrm{Q}_{1}}
$$

As Eq. (7) implies, $\sigma$ is determinate once the boundary condition is given. The behavior of $\sigma$ is portrayed in Fig. 1. When $\sigma>0$, it means the wall heats the fluid up; $\sigma=0$ means the adiabatic wall condition; $\sigma<0$ means the heat in the fluid transfers into the wall. The influence of wall heat flux is obvious at low-speed flows. With the free-stream Mach number increased, the value of $\sigma$ is tending to be constant, i.e. -0.496 , which suggests the proportion of wall heat flux to the viscosity dissipation rate of kinetic energy is to be constant in hypersonic flows.

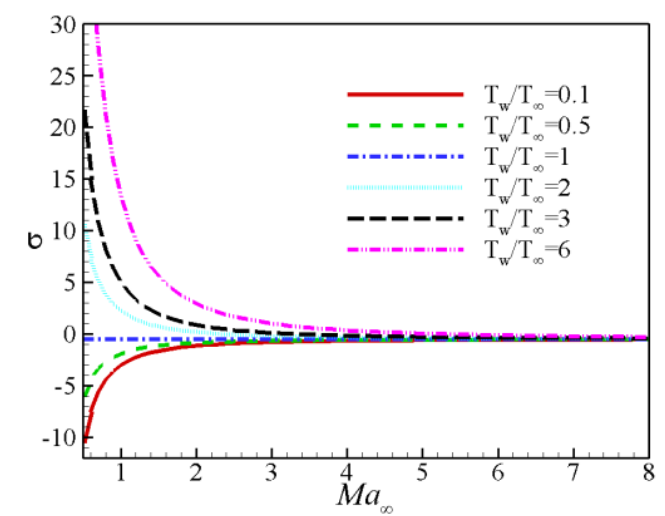

Figure 1. Ratios of wall heat flux along with increased Mach numbers

Assuming $\sigma$ affects the whole boundary layer and its impact is uniform, in the near wall region, the nondimensional entropy increment caused by viscosity dissipation solely could be roughly evaluated by

$$
\Delta s_{\mathrm{vis}}=\frac{1}{\sigma+1}\left(\frac{s}{s_{\infty}}-1\right)
$$

Table 1. Simulation conditions for boundary-layer flows

\begin{tabular}{|c|c|c|c|c|}
\hline & $M a_{\infty}$ & $R e$ & $T_{\mathrm{w}} / T_{\infty}$ & $T_{\mathrm{w}} / T_{\mathrm{r}}$ \\
\hline Case1 & 0.7 & $5.0 \times 10^{4}$ & 1.098 & 1.003 \\
\hline Case2 & 2.25 & $6.35 \times 10^{5}$ & 1.9 & 0.961 \\
\hline Case3 & 6 & $2.0 \times 10^{6}$ & 6.98 & 0.878 \\
\hline
\end{tabular}

\subsection{Process II: Entropy increment ratio}

When the external potential flows pass the vehicle surface, the velocity is gradually decreased to zero due to the viscous frication. Hence, it is natural to foresee that the values of entropy increment near the wall are directly related with inflow speeds. In order to get rid of this dependence on Mach numbers, a new entropy concept, named entropy increment ratio $(\bar{s})$, is introduced.

$$
\bar{s}=\frac{\Delta s_{\mathrm{vis}}}{M a_{\infty}^{2}}
$$

$\bar{s}$ represents the viscous dissipation rate of per unit mechanical energy. We extract corresponding quantities from DNS results to evaluate its character. Table 1 shows the simulation conditions of DNS for flat plates. The incoming flows are subsonic, supersonic and hypersonic, respectively. As aforementioned, the values of $\Delta s_{\text {vis }}$ near the wall are quite different, varying about one order of magnitude from $M a=0.7$ to $M a=6$ (Fig. 2a). On the other hand, $\bar{s}$ exhibits a consistent behavior depicted in Fig. $2 b$. The values near the wall are all falling into the range of 
0.1-0.5, unrelated with Mach numbers. This character is convenient as we always hope a model or physical parameter is general for various flow conditions. Additionally, we could also find that the range where $\bar{s}>0$ is almost equal to the extent where $u / U_{\infty}<1.0$, which means that $\bar{s}$ could be used to discern the boundary-layer for these simple flows.

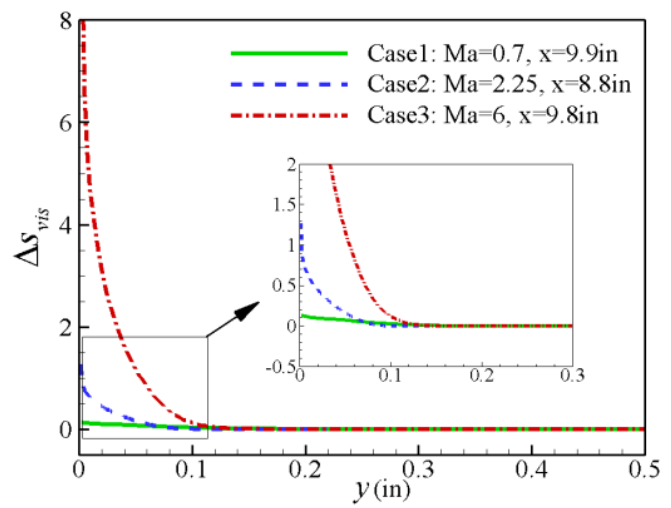

a. Entropy increment profiles normal to the wall

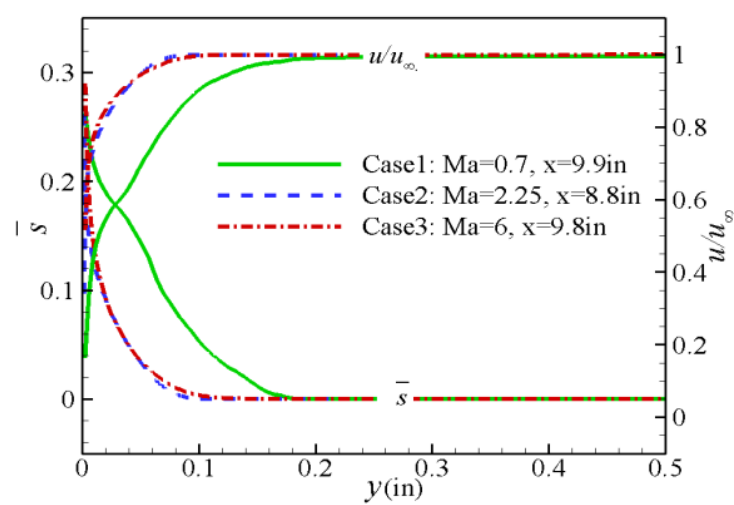

b. Comparison of entropy increment ratio and velocity profiles Figure 2. Entropy and entropy increment ratio profiles normal to the wall of different Mach numbers (DNS results)

\subsection{Process III: Entropy function $f_{\mathrm{s}}$}

After the above two steps, the proposed entropy increment ratio $\bar{s}$ could actually denote the boundarylayers for simple attached flows and even relatively complex separated flows. However, at the external flow region of a typical super/hypersonic flowfield, the values of $\bar{s}$ across shocks or free-shear layers would be in the same magnitude as that in the boundary-layer. To alleviate this inconvenience, we introduce the entropy function $f_{\mathrm{s}}$ by

$$
f_{\mathrm{s}}=1.0-\tanh \left(\bar{s} / l_{\mathrm{s}}^{2}\right)
$$

where $l_{s}$ is the subgrid length-scale, which is designed to be less than 1.0 in the boundary-layer and increase quickly in the external flows. We construct $l_{\mathrm{s}}$ following the spirit of RANS/LES hybrid strategy [11].

$$
l_{\mathrm{s}}=C_{\mathrm{g}} \kappa d_{\mathrm{w}} / C_{\mathrm{DES}} \Delta
$$

in which $C_{\mathrm{g}}=3 \sqrt[3]{\Delta_{x} \Delta_{y} \Delta_{z}} /\left(\Delta_{x}+\Delta_{y}+\Delta_{z}\right)$ is the grid deformation ratio, $\kappa=0.41$ and $C_{\mathrm{DES}}=0.65$ are von
Karman's and Smagorinsky constants respectively, and $\Delta$ is the grid spacing defined by $\Delta=\max (\Delta x, \Delta y, \Delta z) . C_{\mathrm{g}}$ is less than 1.0 in the anisotropy grids near the wall and around 1.0 in the typical LES region. The combination of $\kappa d_{\mathrm{w}}$ represents the Prandtl-van Driest RANS length scale, while $C_{\mathrm{DES}} \Delta$ is the LES subgrid scale employed by DESlike methods.

The character of entropy function $f_{\mathrm{s}}$ is depicted in Fig. 3 , by redrawing Fig. 2 b according to Eq. (10). Compared with the velocity profiles, $f_{\mathrm{s}}$ could denote the boundarylayer with the range $f_{\mathrm{s}}<1.0$ while it exhibit much smoother profiles than those of $\bar{s}$.

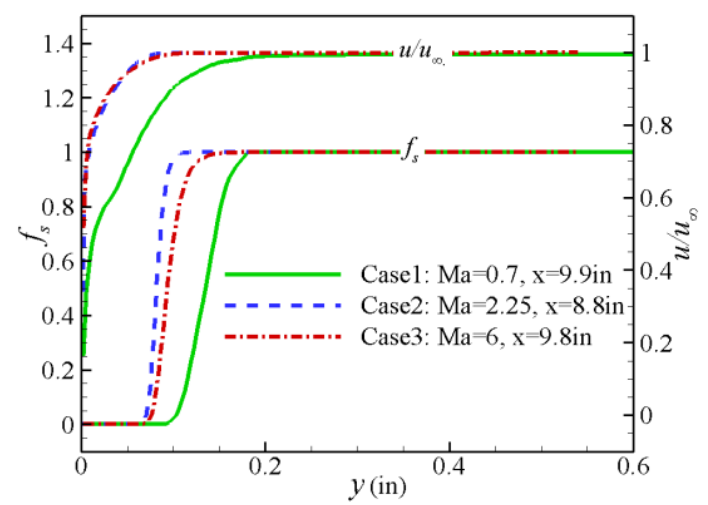

Figure 3. Entropy function $f_{\mathrm{s}}$ profiles normal to the wall (DNS results)

\section{Applications}

By including more test cases, this section provides detailed complements to the derivation of the entropy function $f_{\mathrm{s}}$ and evaluates its performance in complex flows. The governing equations describing the mean flowfield are the time-dependent, compressible Reynoldsaveraged Navier-Stokes equations, along with oneequation Spalart-Allmaras turbulence model. The three dimensional RANS equations are solved by the finite volume method with structured grids and advanced in time by LU-SGS method. The second-order Roe scheme is used for the discretization of the inviscid terms, while the viscous terms are centrally differenced.

\subsection{Low-speed A-airfoil flow $(M a=0.15)$}

As clarified in Process I, the portion of entropy increment caused by the wall heat flux is more prominent in lowspeed flows (Fig. 1). This case is chosen to exhibit the influence of wall temperature and verify the effect of $\sigma$.

The details of inflow conditions and grid construction are available in [12]. The temperature of the incoming flow is $276.48 \mathrm{~K}$ and we prescribe three kinds of wall temperature for comparisons (Table 2). In the cold wall condition, the heat is transferred from the fluid to the wall and the total entropy increment $\Delta s$ becomes negative in the near wall region (Fig. 4a). Comparatively, the hot wall heats the fluid up and $\Delta s$ is much larger than that in the adiabatic wall condition (Fig. $4 b, c$ ).

After removing the effect of wall heat flux by introducing the parameter $\sigma$, the values of entropy increments $\Delta s_{\text {vis }}$ fall to the same level, as can be seen in 
Fig. 5. However, due to the large separation bubble in the hot wall condition, the area where $\Delta s_{\text {vis }}>0$ seems too large to denote the boundary-layer. Nevertheless, with the final Process III, the entropy function $f_{s}$ could predict a proper boundary-layer region (Fig. 6).

Table 2. Three kinds of wall temperature for the A-airfoil

\begin{tabular}{|c|c|c|c|}
\hline Wall conditions & $T_{\mathrm{w}}(\mathrm{K})$ & $T_{\mathrm{w}} / T_{\mathrm{r}}$ & $\sigma$ \\
\hline Cold wall & 100 & 0.36 & -79.34 \\
\hline Hot wall & 1000 & 3.6 & 322.54 \\
\hline Adiabatic wall & 277.68 & 1 & 0 \\
\hline
\end{tabular}
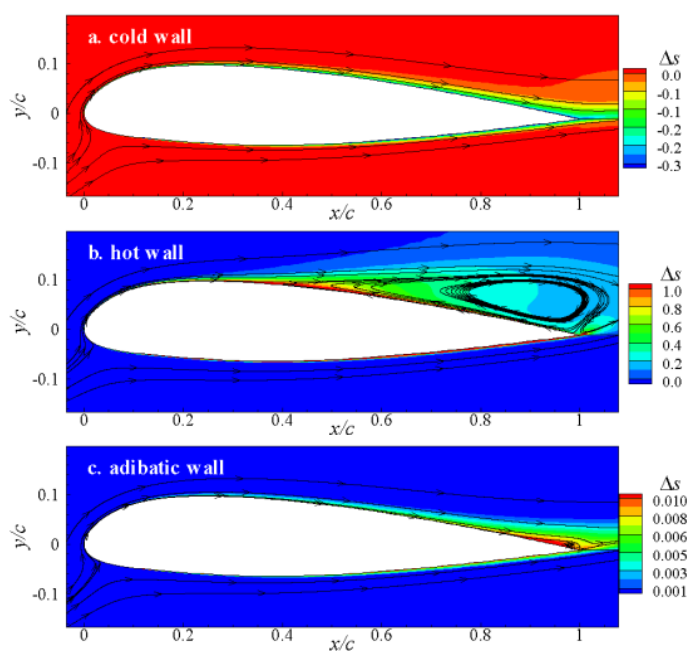

Figure 4. Total entropy increment around the A-airfoil (caused by both viscosity dissipation and wall heat flux)
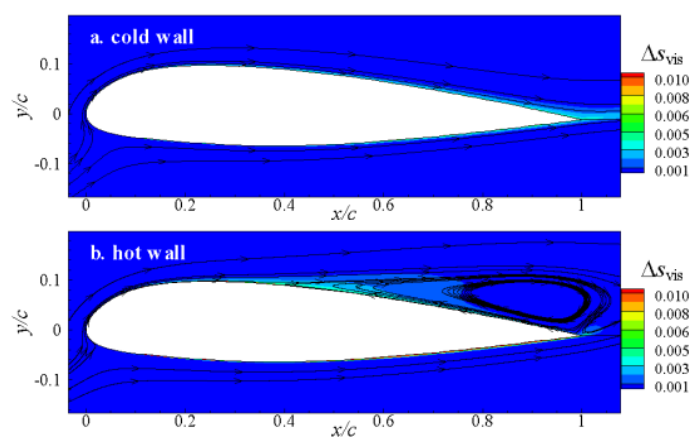

Figure 5. Entropy increment around the A-airfoil (caused by viscosity dissipation only)

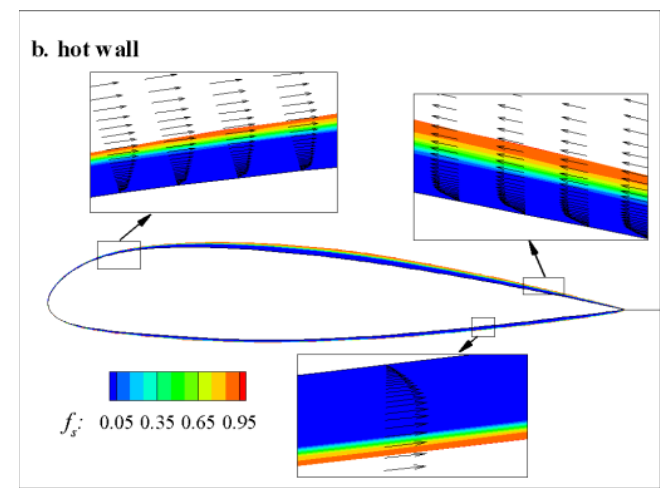

Figure 6. Distributions of entropy function $f_{s}$ and the velocity vectors (only depict $f_{\mathrm{s}}<0.95$ for convenient)

\subsection{Supersonic cavity-ramp flow $(M a=2.92)$}

This case is chosen to evaluate the performance of $f_{s}$ in a typical flowfield. The dominant feature is the supersonic free-shear layer, under which a large recirculation zone fills the whole cavity. On the ramped portion of the cavity, the free-shear layer reattaches to form a turbulent boundary-layer while a compression fan coalesces into a shock wave downstream of the reattachment point, depicted in Fig. 7.

The detailed inflow conditions could be found in [13]. As the wall boundary is adiabatic, we focus on the entropy increments in the external flow.

In this complex flow, entropy is not only increased in the boundary-layer, but in the free-shear layer, the recirculation and across the shocks. To alleviate these unfavorable interactions, the hybrid strategy is adopted through Process III. In general, $f_{\mathrm{s}}$ could detect an acceptable boundary-layer region, compared with the velocity profiles (Fig. 8). But in the cavity where the large recirculation fills, the thickness of the boundarylayer is overestimated by $f_{\mathrm{s}}$. In this area, the value of $\bar{s}$ is almost uniform, and then the distribution of $f_{\mathrm{s}}$ is mainly depending on the local grid resolution according to Eq. (10).

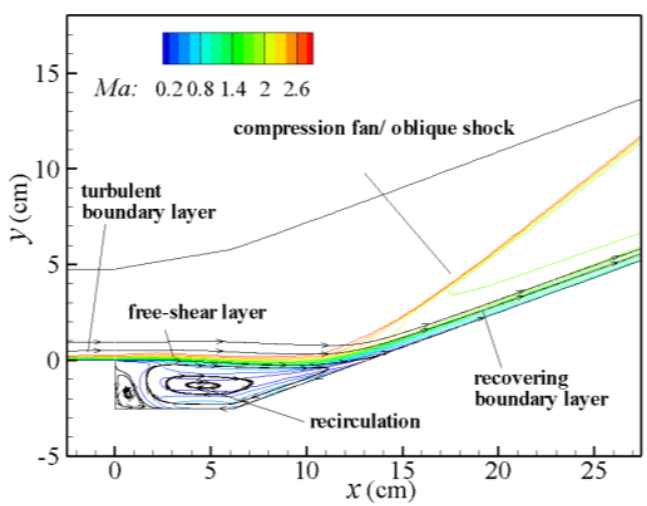

Figure 7. Flow structures for the cavity-ramp (Mach number contours)

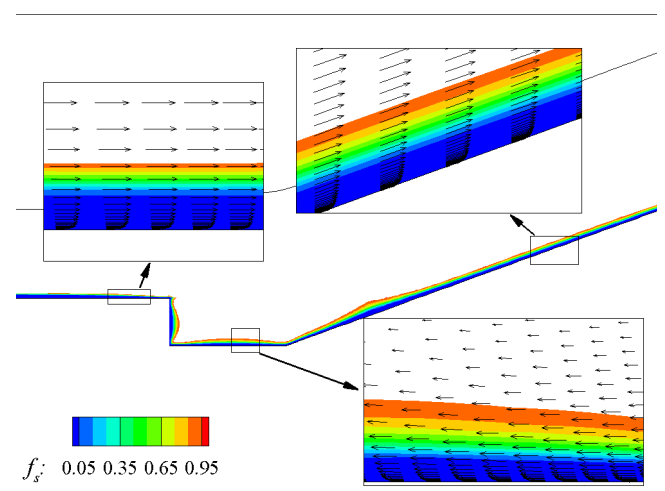

Figure 8. Distributions of fs and the velocity vectors around the cavity-ramp (only depict fs $<0.95$ for convenient)

\subsection{Hypersonic aeroplane flow $(M a=6)$}

For engineering interests, the flow quantities at the edge of the boundary-layer, such as pressure $P_{\mathrm{e}}$ and temperature $T_{\mathrm{e}}$, are essential for thermal shielding design. 
This case is served as an attempt of $f_{\mathrm{s}}$ in denoting the boundary-layer for the engineering application. The flight conditions of this aeroplane configuration are summarized in Table 3. We clustered the grids to all solid surfaces and in the junctions of the body and the wings, ensuring the distance of the first grid line to the wall $y^{+}$is less than 1.0.

As for this configuration, the flow structure is so complex that it is hard to find a sound principle to describe the boundary-layer quantitatively. We choose the streamwise velocity profiles as a compromise. From the comparisons of the three slices at different locations (Fig. 9), the entropy function $f_{\mathrm{s}}$ seems qualified to predict a proper boundary-layer region.

Table 3. Flight conditions of the aeroplane configuration

\begin{tabular}{|c|c|c|c|}
\hline$M a_{\infty}$ & $H(\mathrm{~km})$ & $\alpha\left(^{\circ}\right)$ & $T_{\mathrm{w}}(\mathrm{K})$ \\
\hline 6 & 30 & 0 & 300 \\
\hline
\end{tabular}

* $H$ : flight height; $\alpha$ : angle of attack

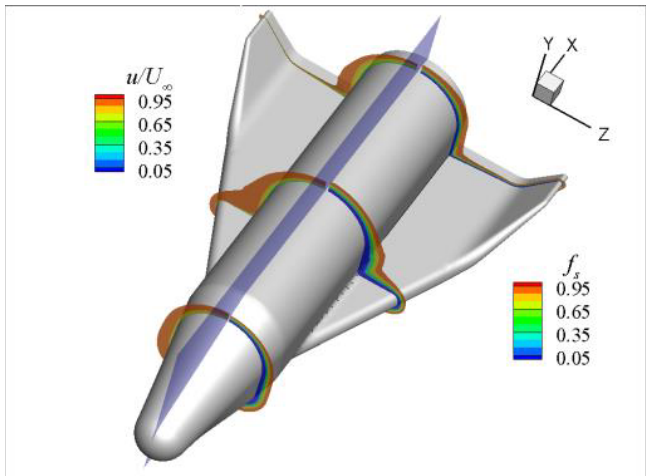

Figure 9. Comparison of boundary-layers denoted by velocity (left, only depict $u / U_{\infty}<0.95$ for convenient) and $f_{\mathrm{s}}$ (right, only depict $f_{\mathrm{s}}<0.95$ for convenient) respectively

\section{Conclusion}

(1) The entropy expression for turbulent boundary-layer is derived and discussed. First, in the near wall region, we remove the effect of wall heat flux through Walz's temperature-velocity relationships. Second, the entropy increment ratio $(\bar{s})$ is introduced with the benefit of Mach number independence, proved by DNS results. Finally, the entropy increment in the external flow is avoided by including the subgrid length-scale $l_{\mathrm{s}}$ according to the hybrid strategy. Then, the entropy increment in the flowfield is only related with the viscosity dissipation in the boundary-layer.
(2) With the above procedures, the proposed entropy function $f_{\mathrm{s}}$ could reliably denote the boundary-layer region for complex flows. However, in the recirculation zone, the performance of $f_{\mathrm{s}}$ is mainly affected by the local grid resolution. This defect is left for our future work.

(3) This entropy function $f_{\mathrm{s}}$ is proposed from the energy aspect, independent on any particular turbulent quantities. Although it cannot be that the absolute best formulation was found to denote the boundary-layer, the present one for $f_{s}$ appears simple and permanent enough to be recommended.

\section{References}

1. G. J. Hu, B. Y. Cao, Z. Y. Guo. Entransy and entropy revisited. Chin Sci Bull, 27, 2974-2977 (2011).

2. G. F. Naterer, J. A. Camberos. The role of entropy and the second law in computational thermofluids. AIAA paper, AIAA-2001-2758, (2001).

3. H. B. Gillian, J. L. Mark. Principle of minimum entropy analysis applied to unsteady flow in hypersonic three-dimensional inlets. AIAA paper, AIAA-2011-2307, (2011).

4. C. E. Shannon. A mathematical theory of communication. Bell Syst Tech J, 27, 379-423, (1948)

5. K. J. Fidkowski, P. L. Roe. Entropy-based mesh refinement, I: the entropy adjoint approach. AIAA paper, AIAA -2009-3790, (2009).

6. D. W. Zaide, P. L. Roe. Entropy-based mesh refinement, II: A New Approach to Mesh Movement. AIAA paper, AIAA-2009-3791, (2009).

7. R. Zhao, C. Yan, J. Yu. A new kind Baldwin-Lomax turbulence model under the limit of entropy. J. Beijing Univ. Aero. Astro, 38(2):175-179, (2012).

8. D. M. McEligot, E. J. Walsh, E. Laurien, et al. Entropy generation in the viscous parts of turbulent boundary layers. J Fluids Engrg, 130:61205, (2008).

9. A. R. Paterson. A First Course in Fluid Dynamics (Cambridge University Press), (1983).

10. A. Walz. Boundary Layers of Flow and Temperature. (MIT Press), (1969).

11. P. R. Spalart. Detached-eddy simulation, Annu. Rev. Fluid Mech., 41, 181-202, (2009).

12. N. Durrani, N. Qin. Behaviour of detached-eddy simulations for mild airfoil trailing-edge separation. J. Aircraft, 48(1), 193-202, (2011).

13. G. S. Settles, D. R. Williams, B. K. Baca, et al. Reattachment of a compressible turbulent free shear layer. AIAA J., 20(1): 60-67, (1982). 